\title{
Blind Source Separation by RBF Neural Network Optimized by GA Peili CONG
}

Information Engineering Department, Liaoning Mechatronics College, Liaoning Dandong, 118009, China

\author{
Congpeili@yeah.net
}

Keywords: Blind Separation; RBF Neural Network; GA; k-Mean Clustering

\begin{abstract}
This work proposed a blind source separation method by RBF neural network optimized by GA, which can improve the separation performance under low SNR condition. The center value and the width value of RBF can be determined by k-mean clustering algorithm and the cost function is set by maximum entropy. For RBF neural network is sensitive to noise, the blind source separation algorithm (BSS) is optimized by GA to obtain the optimal parameters of RBF neural network. This method can implement good separation results under low SNR condition and has better robustness compared with traditional RBF neural network. The computer simulation results show the effectiveness of the proposed method.
\end{abstract}

\section{Introduction}

BSS is a signal processing technology which recovery the independent component from the mixing signal [1]. Recently, BSS has been studied in theory and algorithm and lots of research findings have been reported. Also BSS has been applied in seismic exploration, mobile communication, speech signal processing, array signal processing and biomedical engineering etc [2][3]. Compared with the other BSS algorithms, neural network can be applied not only in linear mixing problems, but also in nonlinear mixing problems. In all kinds of neural network models, $\mathrm{RBF}$ applied in BSS has robust performance. In practical engineering, the problem of complete linearization is very rare. The linear BSS algorithm shows ill-convergence or divergence in nonlinear BSS problems, such as FastICA and JADE [4]. The neural network especially RBF neural network model has strong nonlinear mapping ability. Therefore, neural network BSS algorithm can solve the nonlinear BSS problems. This work studied the RBF neural network BSS algorithm to find the improved method. For the RBF neural network algorithm has the parameters as weights, the center value and the width value, which influence on the performance of the convergence performance for BSS. Now, how to determine these parameters has no effective method. Hereby we proposed a RBF neural network BSS algorithm optimized by GA. The fitness function of GA can be designed according to the cost function of RBF neural network and the real number coding is used to simplify the process. The computer simulation results show the effectiveness of the proposed method.

\section{BSS Model and RBF Neural Network}

Fig. 1 shows the model of the signal and separation diagram [5]. If the signal transmission delay is ignored, the model is the instantaneous mixing. Instantaneous mixing model is the basis of the blind source separation. The blind harmonic signal extraction in chaos can adopt instantaneous mixing model to research. In harmonic signal extraction problem, the harmonic signal mixed with the chaotic signal and the noise is acted as a source signal if the noise meets Gauss distribution. But if the noise is impulse noise, the noise cannot be acted a source signal in the separation algorithm. As a result, we let the noise is adding noise at the output of the mixing process.

In Fig. 1, $\boldsymbol{S}=\left[s_{1}(t), s_{2}(t), \cdots, s_{n}(t)\right]^{\mathrm{T}}$ is unknown and zero mean independent source signals, $\boldsymbol{X}=\left[x_{1}(t), x_{2}(t), \cdots, x_{m}(t)\right]^{\mathrm{T}}$ is the $m$ dimension zero mean random observation vector and 
$\boldsymbol{Y}=\left[y_{1}(t), y_{2}(t), \cdots, y_{n}(t)\right]^{\mathrm{T}}$ is the signals after blind separation. Let the mixing system $\boldsymbol{H}$ be full rank matrix of $m \times n$, the linear mixed process can be given by

$$
\boldsymbol{X}=\boldsymbol{H} \boldsymbol{S}=\sum_{j=1}^{n} h_{j} S_{j}
$$

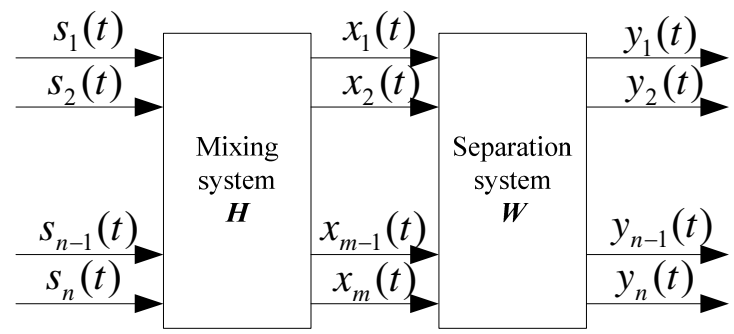

Fig.1 The model of signal mixing and separation

The mixing matrix $\boldsymbol{H}$ is unknown, and the available information is only observed vector $\boldsymbol{X}$. If there is no other information, only $\boldsymbol{X}$ can be used to estimate $\boldsymbol{S}$ and $\boldsymbol{H}$, using FastICA or JADE method is necessarily obtaining more solutions. For the blind harmonic signal extraction under the chaotic background, the source signals consist of different frequency harmonic signals, and the chaotic ones. The source signals can meet the definite solution conditions of ICA. So ICA can solve the problem of the blind harmonic signal extraction. We can construct a separation matrix $W$ which can separate the source signals $\boldsymbol{S}$ from the observed signals $\boldsymbol{X}$, Separation matrix $\boldsymbol{W}$ satisfies [6]

$$
\boldsymbol{Y}=\boldsymbol{W} \boldsymbol{X}=\boldsymbol{W H} \boldsymbol{X}=\boldsymbol{G} \boldsymbol{X}
$$

The aim of blind source separation is to solve the $\boldsymbol{W}$ which let the matrix $\boldsymbol{G}=\boldsymbol{I}$ [7]. In practice, the signal bear the noise interference during the transmission process, so noise must be taken into account for the harmonic signal extraction. RBF neural network is a single hidden layer feedforward neural network model, which has simple structure, nonlinear mapping ability and fast convergence rate. However, if the signal is interfered with noise, the convergence ability of RBF neural network will lead to an unideal result. The basic model of RBF neural network is shown in Fig.2.

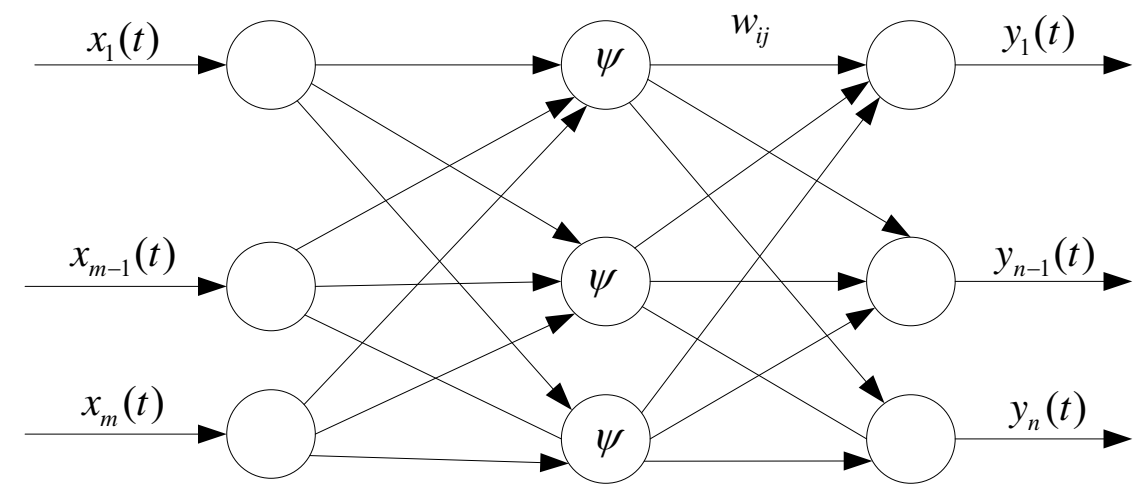

Fig. 2 The basic model of RBF neural network

RBF neural network transfers the nonlinear problem to the linear problem by high dimension space mapping, so it can solve the nonlinear problem with robust convergence performance. RBF neural network with three layers includes input layer, hidden layer and output layer. Let $\boldsymbol{X}=\left[x_{1}(t), x_{2}(t), \cdots, x_{m}(t)\right]^{T}$ is the input vector and $\boldsymbol{Y}=\left[y_{1}(t), y_{2}(t), \cdots, y_{m}(t)\right]^{T}$ is the output vector, and then the RBF neural network can be taken as the separation matrix compared with the model of signal mixing and separation. If the Gaussian function is choose as the transfer function, the RBF neural network can be expressed as follow

$$
g_{h}(x)=\exp \left(-\frac{\left\|x-c_{h}\right\|}{2 \sigma_{h}^{2}}\right) \quad h=1,2, \cdots, n
$$


Where $c_{h}$ is the center point of the Gaussian function at the $h$-th unit of the hidden layer. $\sigma_{i}$ is the normalized parameter at the $h$-th unit of the hidden layer. The output of the RBF neural network can be given by

$$
\boldsymbol{y}=\psi(w g)
$$

Where $\psi($.$) denotes the sigmoid function and w$ is the weights of the RBF neural network. According to the structure of RBF neural network, the output can be rewritten as follow

$$
\begin{aligned}
& \boldsymbol{y}=\psi(\boldsymbol{w u})=\psi(\boldsymbol{w g}(.))=\psi(\boldsymbol{w g}(\boldsymbol{x}, c, \sigma))=\boldsymbol{s} \\
& \boldsymbol{y}=\boldsymbol{s}=\Lambda \boldsymbol{P} \boldsymbol{s}
\end{aligned}
$$

Where $\Lambda$ is the non-singular diagonal matrix and $\boldsymbol{P}$ is the permutation matrix.

The RBF neural network training process includes the center value $c$ and the width value $\sigma$ updating. The center value $c$ can be updated according to simplified rival penalized competitive learning (SRPCL) algorithm. The center value $c$ updating process can be summarized as follow

Step1. Initialization $c(0)$ and the study step size $a$ and $b 0<a<b<1$;

Step2. Compute Eq.7 and Eq.8 as follows

$$
\begin{aligned}
& \beta_{h}(t)=\left\|x(t)-c_{h}(t-1)\right\| \quad h=1,2, \cdots, m \\
& \beta_{k}(t)=\min _{1 \leq h \leq m} \beta_{h}(t) \\
& \beta_{1}(t)=\min _{1 \leq h<m, h \neq k} \beta_{h}(t)
\end{aligned}
$$

Step3. Adjusting the center value $c$ by

$$
\begin{aligned}
& c_{k}(t)=c_{k}(t-1)+a\left(x(t)-c_{k}(t-1)\right) \\
& c_{1}(t)=c_{1}(t-1)+b\left(x(t)-c_{1}(t-1)\right) \\
& c_{h}(t)=c_{h}(t-1) \quad h \neq k, h \neq 1
\end{aligned}
$$

The width value $\sigma$ can be computed by

$$
\sigma=d_{m} / \sqrt{2 m}
$$

After the parameters of the RBF neural network have been determined, the network can be taken as tradition feedforward neural network and be trained by BP algorithm. However, the weights of the neural network can be obtained by the maximum entropy method. The entropy can be given by

$$
H(z)=H(v)+\log |w|+\sum_{i=1}^{n} E\left[\log \psi_{i}\left(y_{i}\right)\right]
$$

Where $|w|=\operatorname{det}(w)$ and

$$
z=\left(\psi_{1}\left(y_{1}\right), \cdots, \psi_{n}\left(y_{n}\right)\right)^{T}
$$

According to Eq. 15 and the RBF model we can obtain

$$
H(v)=H(x)+E[\log (J)]
$$

Where $J$ is the Jacobian matrix of the function $g(x, c, \sigma)$. For $H(v)$ has nothing to do with $w$, the gradient of $w$ can be computed according to stochastic gradient descent algorithm as follow

$$
\begin{aligned}
& \frac{\partial H(z)}{\partial w}=w^{-T}-\phi(y) g^{T} \\
& \phi(y)=-\left(\frac{\psi_{1}^{\prime \prime}\left(y_{1}\right)}{\psi_{1}^{\prime}\left(y_{1}\right)}, \cdots, \frac{\psi_{n}^{\prime \prime}\left(y_{n}\right)}{\psi_{n}^{\prime}\left(y_{n}\right)}\right) \\
& g=g(x, c, \sigma)
\end{aligned}
$$

So the separation matrix can be obtained by using the natural gradient descent algorithm.

$$
\boldsymbol{w}=\boldsymbol{w}+\eta\left(\boldsymbol{I}-\phi(y) y^{T}\right) \boldsymbol{w}
$$

The weights of RBF neural network updating and the maximum entropy algorithm is the same besides the RBF neural network needs computing the center parameter and the width parameter. 
Although RBF neural network can obtain robust convergence performance by gradient descent algorithm with the cost function of maximum entropy, it convergence rate is still slow and the performance will degradation under noise interference. Hereby we use GA optimized the parameters and the weights of the RBF to obtain better convergence performance for BSS.

\section{RBF Neural Network Optimized by GA}

GA is a highly parallel, random and adaptive search method for global optimization. GA Genetic algorithm is a heuristic optimization algorithms proposed by Professor Holland, which is starting from any initial population, by random selection, crossover and mutation, producing a group of individuals more suitable environment to enable groups evolved to be getting better search space area, so multiply constantly evolve from generation to generation, and finally converge to a group of individual the most suitable environmental and obtain the optimal solution of the problem. Genetic algorithm is links with the problem to be solved only encoding and decoding, so it is widely used to solve complex optimization model.

Genetic algorithm takes the fitness function as the evolutionary goal, and only evolves toward the increasing of the value of the fitness function. So the fitness function of GA can set as Eq.16. However, the center parameter and the width parameter of RBF can be taken into account at the same time. Implementation steps for using genetic algorithms to optimize the RBF neural network can be summarized as follows

Step1. Reading the signal and a set of random as an initial value, and the encoding structure of the initial population;

Step2. Computing the fitness value of the individual;

Step3. Competition and choice operation. The sub generation is driven by parent generation compare the individuals according to each fitness value, and the scores $S_{j}$ is obtained by

$$
S_{j}=\left\{\begin{array}{lll}
1 & \text { if } & r<H_{j} /\left(H_{c}+H_{j}\right) \\
0 & \text { if } & r \geq H_{j} /\left(H_{c}+H_{j}\right)
\end{array}\right.
$$

Where $0<r<1$ is a random value, $H_{c}$ and $H_{j}$ are the fitness value of the individual $c$ and $j$.

Step4. Mutation operation. Add a random number in the symbol of all individuals was disturbed, achieve mutation;

Repeat step 2 to step 4 until the maximum number of times set and the optimal solution of the neural network is obtained by the highest individual decoding.

\section{Computer Simulation and Analysis}

In the simulation, three sinusoidal signals with different center frequency as the source signals. The sample frequency of the simulation signal is $f_{s}=2000 \mathrm{~Hz}$, the center frequencies of the sinusoidal signals are $f_{1}=5 \mathrm{~Hz}, f_{2}=20 \mathrm{~Hz}$ and $f_{3}=50 \mathrm{~Hz}$. The noise in the mixed matrix is Gaussian white noise, and the SNR $=10 \mathrm{~dB}$. Randomly generated the mixed matrix by $3 \times 3$ and the mixed signals are shown in Fig.3. The RBF neural network is initialized as follow

$$
\begin{aligned}
& \boldsymbol{w}=\left[\begin{array}{lll}
1 & 0 & 0 \\
0 & 1 & 0 \\
0 & 0 & 1
\end{array}\right] \\
& a=0.0001 \quad b=0.0003
\end{aligned}
$$

The RBF neural network is optimized by GA and the separation results is shown in Fig.4. Fig.4 shows that the proposed method in this paper can obtain ideal separation results. Otherwise, the RBF neural network cannot separate the mixed signals fully under the noise interference, and the best coefficient of the similarity coefficient matrixes is only 0.875 . The proposed method computes the mainly coefficients in the similarity coefficient matrixes all can reach to 0.992 , which shows the effectiveness of the RBF neural network optimized by GA. If the SNR low enough, the RBF neural 
network may counter ill-convergence or divergence problem. However, even the SNR lower than $5 \mathrm{~dB}$, the BSS by RBF neural network optimized by GA can still obtain ideal separation results, which shows its robustness.
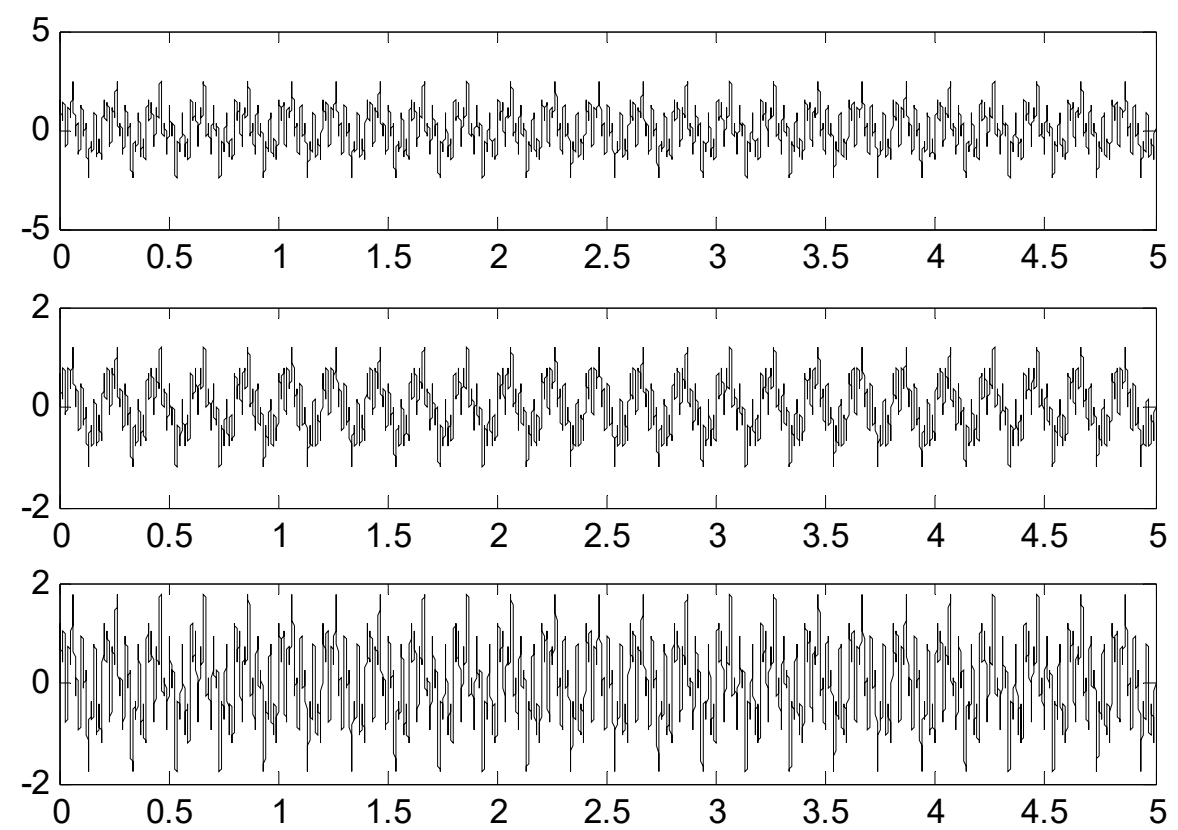

Fig.3 The mixed signal
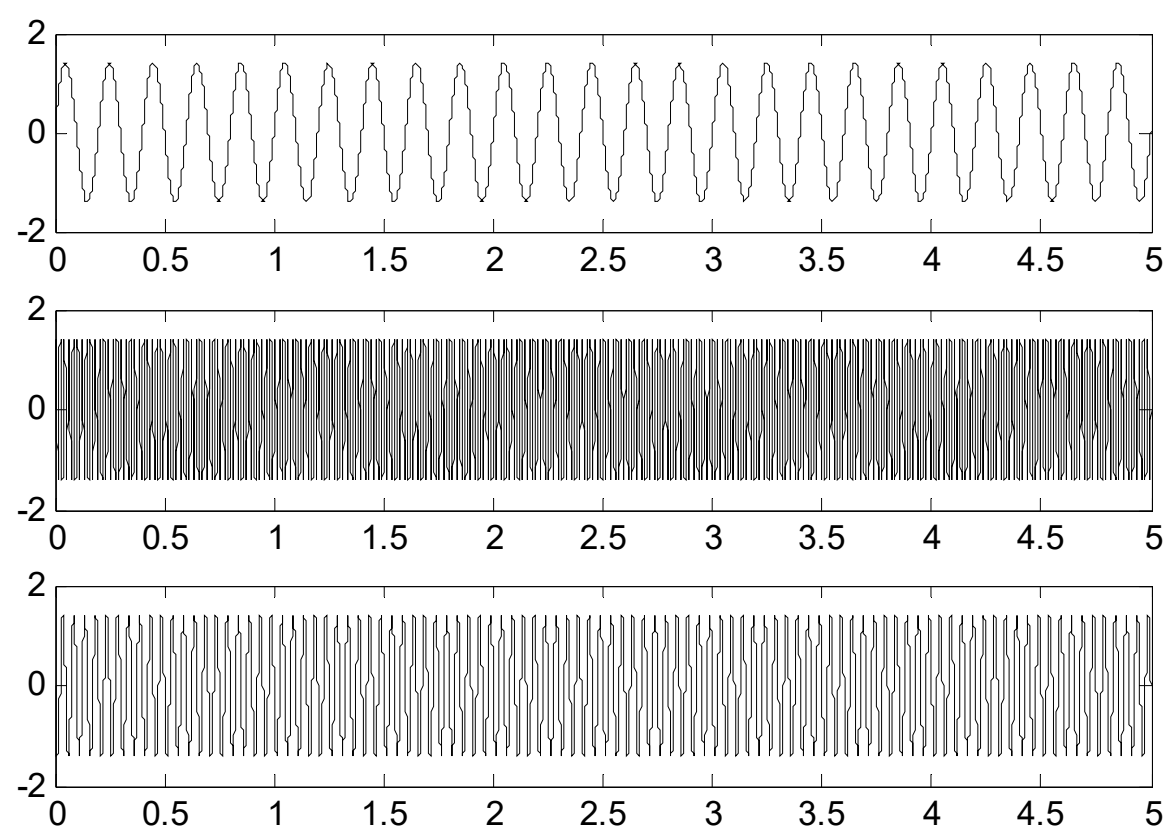

Fig.4 The separation result of RBF optimized by GA

\section{Conclusion}

This work proposed a blind source separation method by RBF neural network optimized by GA, which can improve the RBF neural network performance effectively both in separation results and robustness. The fitness function is used the maximum entropy to construct and the genetic operation uses the traditional GA operation. The computer simulation shows the effectiveness of the proposed method. 


\section{Acknowledgement}

In this paper, the research was sponsored by the Computer network technology training room construction and training project (Project No. JYLX2016007).

\section{References}

[1] Fu Ning, Qiao Li-yan, Peng Xi-yuan. Blind recovery of mixing matrix with sparse sources based on improved k-means clustering and hough transform [J]. Acta Electronica Sinica, 2009, 37(4A): 92-96.

[2] Shindo H, Hirai Y. Blind source separation by a geometrical method[A ]. Proceedings of the International J oint Conference on Neural Networks [C] . Honolulu , HI , USA : IEEE , 2002. $1108-1114$.

[3] Theis F J ,Lang E W, Puntonet C G. A geometric algorithm for overcomplete linear ICA [J]. Neurocomputing, 2004, 56 (124) : 381 - 398.

[4] Cui Peng-hui, Xiong Tao, Jiang Hua, et al. RSDFF blind separation algorithm based on decision feedback-feedforward [J]. Acta Electronica Sinica, 2016, 44(3): 633-638.

[5] Tu Shi-long, Zheng Hui, Single channel blind separation of digital modulate signals with close carriers and different data rates [J]. Journal of Circuits and Systems, 2010, 15(3): 43-47.

[6] Du Jian, Gong Ke-xian, Peng Hua. Algorithm for blind separation of PCMA based on CHASE decoding [J]. Journal on Communication, 2015, 36(3): 1-6.

[7] GAO B, WOO W L, DLAY S S. Unsupervised single-channel separation of nonstationary signals using gammatone filterbank and itakura-saito nonnegative matrix two-dimensional factorizations [J]. IEEE Trans on Circuits and Systems, 2013, 60(3): 662-675. 